\title{
Monomial Crystals and Partition Crystals
}

Peter TINGLEY

Department of Mathematics, Massachusetts Institute of Technology,

77 Massachusetts Avenue, Cambridge, MA 02139, USA

E-mail: ptingley@math.mit.edu

URL: http://www-math.mit.edu/ ptingley/

Received February 10, 2010, in final form April 12, 2010; Published online April 21, 2010

doi:10.3842/SIGMA.2010.035

\begin{abstract}
Recently Fayers introduced a large family of combinatorial realizations of the fundamental crystal $B\left(\Lambda_{0}\right)$ for $\widehat{\mathfrak{s l}}_{n}$, where the vertices are indexed by certain partitions. He showed that special cases of this construction agree with the Misra-Miwa realization and with Berg's ladder crystal. Here we show that another special case is naturally isomorphic to a realization using Nakajima's monomial crystal.
\end{abstract}

Key words: crystal basis; partition; affine Kac-Moody algebra

2010 Mathematics Subject Classification: 17B37; 05E10

\section{Introduction}

Fix $n \geq 3$ and let $B\left(\Lambda_{0}\right)$ be the crystal corresponding to the fundamental representation of $\widehat{\mathfrak{s l}}_{n}$. Recently Fayers [2] constructed an uncountable family of combinatorial realizations of $B\left(\Lambda_{0}\right)$, all of whose underlying sets are indexed by certain partitions. Most of these are new, although two special cases have previously been studied. One is the well known Misra-Miwa realization [12]. The other is the ladder crystal developed by Berg [1].

The monomial crystal was introduced by Nakajima in [13, Section 3] (see also [5, 10]). Nakajima considers a symmetrizable Kac-Moody algebra whose Dynkin diagram has no odd cycles, and constructs combinatorial realizations for the crystals of all integrable highest weight modules. In the case of the fundamental crystal $B\left(\Lambda_{0}\right)$ for $\widehat{\mathfrak{s l}}_{n}$, we shall see that the construction works exactly as stated in all cases, including $n$ odd when there is an odd cycle.

Here we construct an isomorphism between a realization of $B\left(\Lambda_{0}\right)$ using Nakajima's monomial crystal and one case of Fayers' partition crystal. Of course any two realizations of $B\left(\Lambda_{0}\right)$ are isomorphic, so the purpose is not to show that the two realizations are isomorphic, but rather to give a simple and natural description of that isomorphism.

This article is organized as follows. Sections 2, 3 and 4 review necessary background material. Section 5 contains the statement and proof of our main result. In Section 6 we briefly discuss some questions arising from this work.

\section{Crystals}

In Sections 3 and 4 we review the construction of Nakajima's monomial crystals and Fayers' partition crystals. We will not assume the reader has any prior knowledge of these constructions. We will however assume that the reader is familiar with the notion of a crystal, so will only provide enough of an introduction to that subject to fix conventions, and refer the reader to [9] or [6] for more details.

We only consider crystals for the affine Kac-Moody algebra $\widehat{\mathfrak{s l}}_{n}$. For us, an $\widehat{\mathfrak{s l}}_{n}$ crystal is the crystal associated to an integrable highest weight $\widehat{\mathfrak{s l}}_{n}$ module. It consists of a set $B$ along with 
operators $\tilde{e}_{\bar{\imath}}, \tilde{f}_{\bar{\imath}}: B \rightarrow B \cup\{0\}$ for each $\bar{\imath}$ modulo $n$, which satisfy various axioms. Often the definition of a crystal includes three functions wt, $\varphi, \varepsilon: B \rightarrow P$, where $P$ is the weight lattice. In the case of crystals of integrable modules, these functions can be recovered (up shifting in a null direction) from the knowledge of the $\tilde{e}_{\bar{\imath}}$ and $\tilde{f}_{\bar{\imath}}$, so we will not count them as part of the data.

\section{The monomial crystal}

This construction was first introduced in [13, Section 3], where it is presented for symmetrizable Kac-Moody algebras where the Dynkin diagram has no odd cycles. In particular, it only works for $\widehat{\mathfrak{s l}}_{n}$ when $n$ is even. However, in Section 5 we show that for the fundamental crystal $B\left(\Lambda_{0}\right)$ the most naive generalization to the case of odd $n$ gives rise to the desired crystal, so the results in this note hold for all $n \geq 3$.

We now fix some notation, largely following [13, Section 3].

- Let $\widetilde{I}$ be the set of pairs $(\bar{l}, k)$ where $\bar{\imath}$ is a residue $\bmod n$ and $k \in \mathbb{Z}$.

- Define commuting formal variables $Y_{\bar{\imath}, k}$ for all pairs $(\bar{\imath}, k) \in \widetilde{I}$.

- Let $\mathcal{M}$ be the set of monomials in the variables $Y_{\bar{\imath}, k}^{ \pm 1}$. To be precise, a monomial $m \in \mathcal{M}$ is a product $\prod_{(\bar{\imath}, k) \in \widetilde{I}} Y_{\bar{\imath}, k}^{u_{\bar{i}, k}}$ with all $u_{\bar{\imath}, k} \in \mathbb{Z}$ and $u_{\bar{\imath}, k}=0$ for all but finitely many $(\bar{\imath}, k) \in \widetilde{I}$.

- For each pair $(\bar{\imath}, k) \in \widetilde{I}$, let $A_{\bar{\imath}, k}=Y_{\bar{\imath}, k-1} Y_{\bar{\imath}, k+1} Y_{\bar{\imath}+\overline{1}, k}^{-1} Y_{\bar{\imath}-\overline{1}, k}^{-1}$.

- Fix a monomial $m=\prod_{(\bar{\imath}, n) \in \widetilde{I}} Y_{\bar{\imath}, k}^{u_{\bar{\imath}, k}} \in \mathcal{M}$. For $L \in \mathbb{Z}$ and $\bar{\imath} \in \mathbb{Z} / n \mathbb{Z}$, define:

$$
\begin{aligned}
& \operatorname{wt}(m):=\sum_{(\bar{\imath}, k) \in \widetilde{I}} u_{\bar{\imath}, k} \Lambda_{\bar{\imath}}, \quad \varepsilon_{\bar{\imath}, L}(m):=-\sum_{l \geq L} u_{\bar{\imath}, l}(m), \quad \varphi_{\bar{\imath}, L}(m):=\sum_{l \leq L} u_{\bar{\imath}, l}(m), \\
& \varepsilon_{\bar{\imath}}(m):=\max \left\{\varepsilon_{\bar{\imath}, L}(m) \mid L \in \mathbb{Z}\right\}, \quad p_{\bar{\imath}}(m):=\max \left\{L \in \mathbb{Z} \mid \varepsilon_{\bar{\imath}, L}(m)=\varepsilon_{\bar{\imath}}(m)\right\}, \\
& \varphi_{\bar{\imath}}(m):=\max \left\{\varphi_{\bar{\imath}, L}(m) \mid L \in \mathbb{Z}\right\}, \quad q_{\bar{\imath}}(m):=\min \left\{L \in \mathbb{Z} \mid \varphi_{\bar{\imath}, L}(m)=\varphi_{\bar{\imath}}(m)\right\} .
\end{aligned}
$$

Note that one always has $\varphi_{\bar{\imath}}(m), \varepsilon_{\bar{\imath}}(m) \geq 0$. Furthermore, if $\varepsilon_{\bar{\imath}}(m)>0$ then $p_{\bar{\imath}}$ is finite, and if $\varphi_{\bar{\imath}}(m)>0$ then $q_{\bar{\imath}}(m)$ is finite.

- Define $\tilde{e}_{\bar{\imath}}^{M}, \tilde{f}_{\bar{\imath}}^{M}: \mathcal{M} \cup\{0\} \rightarrow \mathcal{M} \cup\{0\}$ for each residue $\bar{\imath}$ modulo $n$ by $\tilde{e}_{\bar{\imath}}^{M}(0)=\tilde{f}_{\bar{\imath}}^{M}(0)=0$,

$$
\tilde{e}_{\bar{\imath}}^{M}(m):=\left\{\begin{array}{ll}
0 & \text { if } \varepsilon_{\bar{\imath}}(m)=0, \\
A_{\bar{\imath}, p_{\bar{\imath}}(m)-1} m & \text { if } \varepsilon_{\bar{\imath}}(m)>0,
\end{array} \quad \tilde{f}_{\bar{\imath}}^{M}(m):= \begin{cases}0 & \text { if } \varphi_{\bar{\imath}}(m)=0, \\
A_{\bar{\imath}, q_{\bar{\imath}}(m)+1}^{-1} m & \text { if } \varphi_{\bar{\imath}}(m)>0 .\end{cases}\right.
$$

Definition 3.1. A monomial $m$ is called dominant if $u_{\bar{\imath}, k} \geq 0$ for all $(\bar{\imath}, k) \in \widetilde{I}$.

Definition 3.2. Assume $n$ is even. Then $m$ is called compatible if $u_{\bar{\imath}, k} \neq 0$ implies $k \cong \bar{\imath}$ modulo 2 .

Definition 3.3. Let $\mathcal{M}(m)$ be the set of monomials in $\mathcal{M}$ which can be reached from $m$ by applying various $\tilde{e}_{\bar{\imath}}^{M}$ and $\tilde{f}_{\bar{\imath}}^{M}$.

Theorem 3.4 ([13, Theorem 3.1]). Assume $n>2$ is even, and let $m$ be a dominant, compatible monomial. Then $\mathcal{M}(m)$ along with the operators $\tilde{e}_{\bar{\imath}}^{M}$ and $\tilde{f}_{\bar{\imath}}^{M}$ is isomorphic to the crystal $B(\operatorname{wt}(m))$ of the integrable highest weight $\widehat{\mathfrak{s l}}_{n}$ module $V(\operatorname{wt}(m))$.

Remark 3.5. Notice that although Theorem 3.4 only holds when $n$ is even, the operators $\tilde{e}_{\bar{\imath}}^{M}$ and $\tilde{f}_{\bar{\imath}}^{M}$ are well defined for any $n \geq 3$ and any monomial $m$. When $n$ is odd or $m$ does not satisfy the conditions of Theorem $3.4, \mathcal{M}(m)$ need not be a crystal. However, as we prove in Section 5, even when $n$ is odd $\mathcal{M}\left(Y_{\overline{0}, 0}\right)$ is a copy of the crystal $B\left(\Lambda_{0}\right)$ of the fundamental representation of $\widehat{\mathfrak{s l}}_{n}$. 


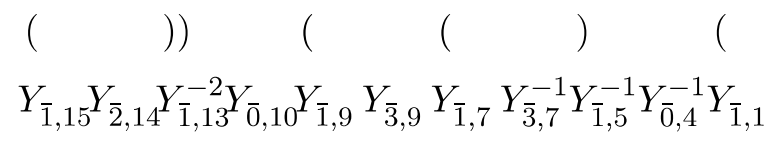

Figure 1. The operators $\tilde{e}_{\bar{\imath}}^{M}$ and $\tilde{f}_{\bar{\imath}}^{M}$ on a monomial $m \in \mathcal{M}$ for $n=4$. We calculate $\widetilde{e}_{\overline{1}}^{M}$ and $\widetilde{f}_{\overline{1}}^{M}$. The factors $Y_{\bar{\imath}, k}$ of $m$ are arranged from left to right by decreasing $k$. The string of brackets $S_{\overline{1}}(m)$ is as shown above the monomial. The first uncanceled ")" from the right corresponds to a factor of $Y_{\overline{1}, 13}^{-1}$. Thus $\widetilde{e}_{\overline{1}}^{M}(m)=A_{\overline{1}, 12} m=Y_{\overline{1}, 11} Y_{\overline{1}, 13} Y_{\overline{0}, 12}^{-1} Y_{\overline{2}, 12}^{-1} m$. The first uncanceled "(" from the left corresponds to a factor of $Y_{\overline{1}, 9}$, so $\widetilde{f}_{\overline{1}}^{M}(m)=A_{\overline{1}, 10}^{-1} m=Y_{\overline{1}, 9}^{-1} Y_{\overline{1}, 11}^{-1} Y_{\overline{0}, 10} Y_{\overline{2}, 10} m$.

We find it convenient to use the following slightly different but equivalent definition of $\tilde{e}_{\bar{\imath}}^{M}$ and $\tilde{f}_{\bar{\imath}}^{M}$. For each $\bar{\imath}$ modulo $n$, let $S_{\bar{\imath}}(m)$ be the string of brackets which contains a "(" for every factor of $Y_{\bar{\imath}, k}$ in $m$ and a ")" for every factor of $Y_{\bar{\imath}, k}^{-1} \in m$, for all $k \in \mathbb{Z}$. These are ordered from left to right in decreasing order of $k$, as shown in Fig. 1. Cancel brackets according to usual conventions, and set

$$
\begin{aligned}
& \tilde{e}_{\bar{\imath}}^{M}(m)= \begin{cases}0 & \text { if there is no uncanceled ")" in } m, \\
A_{\bar{\imath}, k-1} m & \text { if the first uncanceled ")" from the right comes from a factor } \left.Y_{\bar{\imath}, k}^{-1}, 2\right)\end{cases} \\
& \tilde{f}_{\bar{\imath}}^{M}(m)= \begin{cases}0 & \text { if there is no uncanceled "(" in } m, \\
A_{\bar{\imath}, k+1}^{-1} m & \text { if the first uncanceled "(" from the left comes from a factor } Y_{\bar{\imath}, k} .\end{cases}
\end{aligned}
$$

It is a straightforward exercise to see that the operators defined in (2) agree with those in (1).

\section{Fayers' crystal structures}

\subsection{Partitions}

A partition $\lambda$ is a finite length non-increasing sequence of positive integers. Associated to a partition is its Ferrers diagram. We draw these diagrams as in Fig. 2 so that, if $\lambda=\left(\lambda_{1}, \ldots, \lambda_{N}\right)$, then $\lambda_{i}$ is the number of boxes in row $i$ (rows run southeast to northwest). Let $\mathcal{P}$ denote the set of all partitions. For $\lambda, \mu \in \mathcal{P}$, we say $\lambda$ is contained in $\mu$ if the diagram for $\lambda$ fits inside the diagram for $\mu$.

Fix $\lambda \in \mathcal{P}$ and a box $b$ in (the diagram of) $\lambda$. We now define several statistics of $b$. See Fig. 2 for an example illustrating these. The coordinates of $b$ are the coordinates $\left(x_{b}, y_{b}\right)$ of the center of $b$, using the axes shown in Fig. 2. The content $c(b)$ is $y_{b}-x_{b}$. The arm length of $b$ is $\operatorname{arm}(b):=\lambda_{x_{b}+1 / 2}-y_{b}-1 / 2$, where $\lambda_{x_{b}+1 / 2}$ is the length of the row through $b$. The hook length of $b$ is $\operatorname{hook}(b):=\lambda_{x_{b}+1 / 2}-y_{b}+\lambda_{y_{b}+1 / 2}^{\prime}-x_{b}$, where $\lambda_{x_{b}+1 / 2}$ is the length of the row containing $b$ and $\lambda_{y_{b}+1 / 2}^{\prime}$ is the length of the column containing $b$.

\subsection{The general construction}

We now recall Fayers' construction [2] of the crystal $B_{\Lambda_{0}}$ for $\widehat{\mathfrak{s l}}_{n}$ in its most general version. We begin with some notation. An arm sequence is a sequence $A=A_{1}, A_{2}, \ldots$ of integers such that

(i) $t-1 \leq A_{t} \leq(n-1) t$ for all $t \geq 1$, and

(ii) $A_{t+u} \in\left[A_{t}+A_{u}, A_{t}+A_{u}+1\right]$ for all $t, u \geq 1$.

Fix an arm sequence $A$. A box $b$ in a partition $\lambda$ is called $A$-illegal if, for some $t \in \mathbb{Z}_{>0}$, $\operatorname{hook}(b)=n t$ and $\operatorname{arm}(b)=A_{t}$. A partition $\lambda$ is called $A$-regular if it has no $A$-illegal boxes. Let $B^{A}$ denote the set of $A$-regular partitions. 


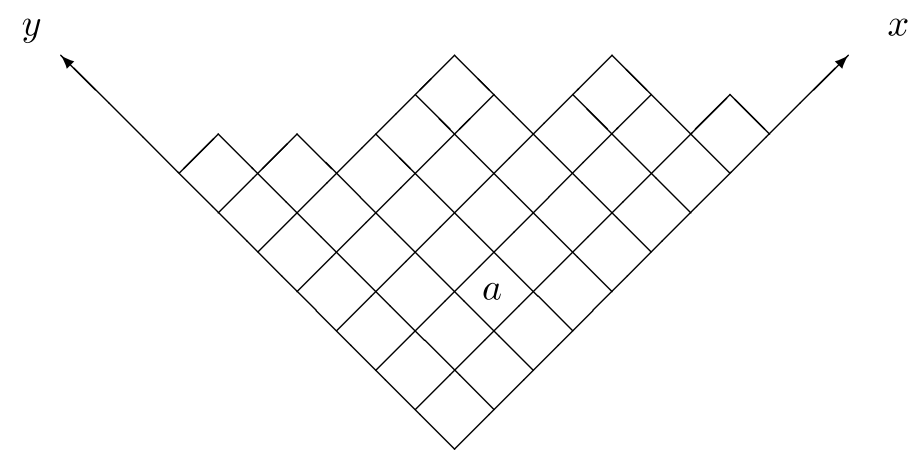

$\begin{array}{llllllllllll}\cdots & 9 & 8 & 7 & 6 & 5 & 4 & 3 & 2 & 1 & 0-1-2-3-4-5-6-7-8-9 & \cdots\end{array}$

Figure 2. The partition $\lambda=(7,6,5,5,5,3,3,1)$, drawn in "Russian" notation. The parts $\lambda_{i}$ are the lengths of the "rows" of boxes sloping southeast to northwest. The center of each box $b$ has coordinate $\left(x_{b}, y_{b}\right)$ for some $x_{b}, y_{b} \in \mathbb{Z}+1 / 2$. For the box labeled $a, x_{a}=2.5$ and $y_{a}=1.5$. The content $c(a)=y_{a}-x_{a}$ records the horizontal position of $a$, reading right to left. In this case, $c(a)=-1$. Other relevant statistics are $\operatorname{hook}(a)=8, \operatorname{arm}(a)=3$ and $h(a)=4$. This partition is not in $B^{H}$ for $\widehat{\mathfrak{s l}}_{4}$, since the box $a$ is $A^{H_{-}}$ illegal.

For $\lambda \in \mathcal{P}$ and a box $b \in \lambda$. The color of $b$ is the residue $\bar{c}(b)$ of $c(b)$ modulo $n$, where as in Section 4.1, $c(b)$ is the content of $b$. See Fig. 3.

Fix $\lambda \in \mathcal{P}$ and a residue $\bar{\imath}$ modulo $n$. Define

- $A(\lambda)$ is the set of boxes $b$ which can be added to $\lambda$ so that the result is still a partition.

- $R(\lambda)$ is the set of boxes $b$ which can be removed from $\lambda$ so that the result is still a partition.

- $A_{\bar{\imath}}(\lambda)=\{b \in A(\lambda)$ such that $\bar{c}(b)=\bar{\imath}\}$.

- $R_{\bar{\imath}}(\lambda)=\{b \in R(\lambda)$ such that $\bar{c}(b)=\bar{\imath}\}$.

For each partition $\lambda$ and each arm sequence $A$, define a total order $\succ_{A}$ on $A_{\bar{\imath}}(\lambda) \cup R_{\bar{\imath}}(\lambda)$ as follows. Fix $b=(x, y), b^{\prime}=\left(x^{\prime}, y^{\prime}\right) \in A_{\bar{\imath}}(\lambda) \cup R_{\bar{\imath}}(\lambda)$, and assume $b \neq b^{\prime}$. Then there is some $t \in \mathbb{Z} \backslash\{0\}$ such $c\left(b^{\prime}\right)-c(b)=n t$. Interchanging $b$ and $b^{\prime}$ if necessary, assume $t>0$. Define $b^{\prime} \succ_{A} b$ if $y^{\prime}-y>A_{t}$, and $b \succ_{A} b^{\prime}$ otherwise. It follows from the definition of an arm sequence that $\succ_{A}$ is transitive.

Fix a partition $\lambda$. Construct a string of brackets $S_{\bar{\imath}}^{A}(\lambda)$ by placing a "(" for every $b \in A_{\bar{\imath}}(\lambda)$ and a ")" for every $b \in R_{\bar{\imath}}(\lambda)$, in decreasing order from left to right according to $\succ_{A}$. Cancel brackets according to the usual rule. Define maps $\tilde{e}_{\bar{\imath}}^{A}, \tilde{f}_{\bar{\imath}}^{A}: \mathcal{P} \cup\{0\} \rightarrow \mathcal{P} \cup\{0\}$ by $e_{\bar{\imath}}^{A}(0)=f_{\bar{\imath}}^{A}(0)=0$,

$$
\begin{aligned}
& \tilde{e}_{\bar{\imath}}^{A}(\lambda)= \begin{cases}\lambda \backslash b & \text { if the first uncanceled ")" from the right in } S_{\bar{\imath}}^{A} \text { corresponds to } b, \\
0 & \text { if there is no uncanceled ")" in } S_{\bar{\imath}}^{A},\end{cases} \\
& \tilde{f}_{\bar{\imath}}^{A}(\lambda)= \begin{cases}\lambda \sqcup b & \text { if the first uncanceled "(" from the left in } S_{\bar{\imath}}^{A} \text { corresponds to } b, \\
0 & \text { if there is no uncanceled "(" in } S_{\bar{\imath}}^{A} .\end{cases}
\end{aligned}
$$

Theorem 4.1 ([2, Theorem 2.2]). Fix $n \geq 3$ and an arm sequence $A$. Then $B^{A} \cup\{0\}$ is preserved by the maps $\tilde{e}_{\bar{\imath}}^{A}$ and $\tilde{f}_{\bar{\imath}}^{A}$, and forms a copy of the crystal $B\left(\Lambda_{0}\right)$ for $\widehat{\mathfrak{s l}}_{n}$, where as above $B^{A}$ is the set of partitions with no A-illegal hooks.

Remark 4.2. The operators $\tilde{e}_{\bar{\imath}}$ and $\tilde{f}_{\bar{\imath}}$ are defined on all partitions. However, as noted in [2], the component generated by a non $A$-regular partition need not be an $\widehat{\mathfrak{s l}}_{n}$ crystal. 


\subsection{Special case: the horizontal crystal}

Consider the case of the construction given in Section 4.2 where, for all $t, A_{t}=\lceil n t / 2\rceil-1$ (it is straightforward to see that this satisfies the definition of an arm sequence). This arm sequence will be denoted $A^{H}$. For convenience, we denote $B^{A^{H}}$ simply by $B^{H}$ and the operators $\tilde{e}_{\bar{\imath}}^{A^{H}}$ and $\tilde{f}_{\bar{\imath}}^{A^{H}}$ from Section 4.2 by $\tilde{e}_{\bar{\imath}}^{H}$ and $\tilde{f}_{\bar{\imath}}^{H}$.

Definition 4.3. Let $b=(x, y)$ be a box. The height of $b$ is $h(b):=x+y$.

Lemma 4.4. Fix $\lambda \in B^{H}$, and let $b, b^{\prime} \in A_{\bar{\imath}}(\lambda) \cup R_{\bar{\imath}}(\lambda)$ with $b \neq b^{\prime}$. Then $b^{\prime} \succ_{A^{H}} b$ if and only if

(i) $h\left(b^{\prime}\right)>h(b)$, or

(ii) $h\left(b^{\prime}\right)=h(b)$ and $c\left(b^{\prime}\right)>c(b)$.

Proof. Since $\bar{c}(b)=\bar{c}\left(b^{\prime}\right)$, we have $c\left(b^{\prime}\right)-c(b)=n t$ for some $t \in \mathbb{Z} \backslash\{0\}$. First consider the case when $t>0$. Then by definition, $b^{\prime} \succ_{A^{H}} b$ if and only if

$$
y^{\prime}-y>A_{t}^{H},
$$

where

$$
\begin{aligned}
A_{t}^{H} & =\lceil n t / 2\rceil-1=\left\lceil\left(c\left(b^{\prime}\right)-c(b)\right) / 2\right\rceil-1=\left\lceil\left(y^{\prime}-x^{\prime}-y+x\right) / 2\right\rceil-1 \\
& = \begin{cases}\left(y^{\prime}-x^{\prime}-y+x\right) / 2-1 & \text { if } y^{\prime}-x^{\prime}-y+x \text { is even, } \\
\left(y^{\prime}-x^{\prime}-y+x\right) / 2-1 / 2 & \text { if } y^{\prime}-x^{\prime}-y+x \text { is odd. }\end{cases}
\end{aligned}
$$

Since $y^{\prime}-y \in \mathbb{Z},(4)$ holds if and only if $y^{\prime}-y>\left(y^{\prime}-x^{\prime}-y+x\right) / 2-1 / 2$, which rearranges to $h\left(b^{\prime}\right) \geq h(b)$.

The case $t<0$ follows immediately from the case $t>0$ since $\succ_{A^{H}}$ is a total order.

Lemma 4.4 implies that $\tilde{e}_{\bar{\imath}}^{H}$ and $\tilde{f}_{\bar{\imath}}^{H}$ are as described as in Fig. 3.

\section{A crystal isomorphism}

Define a map $\Psi: \mathcal{P} \cup\{0\} \rightarrow \mathcal{M} \cup\{0\}$ by $\Psi(0)=0$, and, for all $\lambda \in \mathcal{P}$,

$$
\Psi(\lambda):=\prod_{b \in A(\lambda)} Y_{\bar{c}(b), h(b)-1} \prod_{b \in R(\lambda)} Y_{\bar{c}(b), h(b)+1}^{-1}
$$

Here $A(\lambda)$ and $R(\lambda)$ are as in Section 4.2.

Theorem 5.1. For any $n \geq 3$, any $\bar{\imath}$ modulo $n$, and any $\lambda \in B^{H}$, we have $\Psi\left(\tilde{e}_{\bar{\imath}}^{H} \lambda\right)=\tilde{e}_{\bar{\imath}}^{M} \Psi(\lambda)$ and $\Psi\left(\tilde{f}_{\bar{\imath}}^{H} \lambda\right)=\tilde{f}_{\bar{\imath}}^{M} \Psi(\lambda)$, where $\tilde{e}_{\bar{\imath}}^{M}, \tilde{f}_{\bar{\imath}}^{M}$ are as in Section 3 and $\tilde{e}_{\bar{\imath}}^{H}, \tilde{f}_{\bar{\imath}}^{H}$ are as in Section 4.3 .

Before proving Theorem 5.1 we will need a few technical lemmas.

Lemma 5.2. Let $\lambda$ and $\mu$ be partitions such that $\mu=\lambda \sqcup b$ for some box $b$. Then $\Psi(\mu)=$ $A_{\bar{c}(b), h(b)}^{-1} \Psi(\lambda)$.

Proof. Let $i=\bar{c}(b)$. It is clear that the pair $(A(\lambda), R(\lambda))$ differs from $(A(\mu), R(\mu))$ in exactly the following four ways:

- $b \in A_{\bar{\imath}}(\lambda) \backslash A_{\bar{\imath}}(\mu)$.

- $b \in R_{\bar{\imath}}(\mu) \backslash R_{\bar{\imath}}(\lambda)$. 


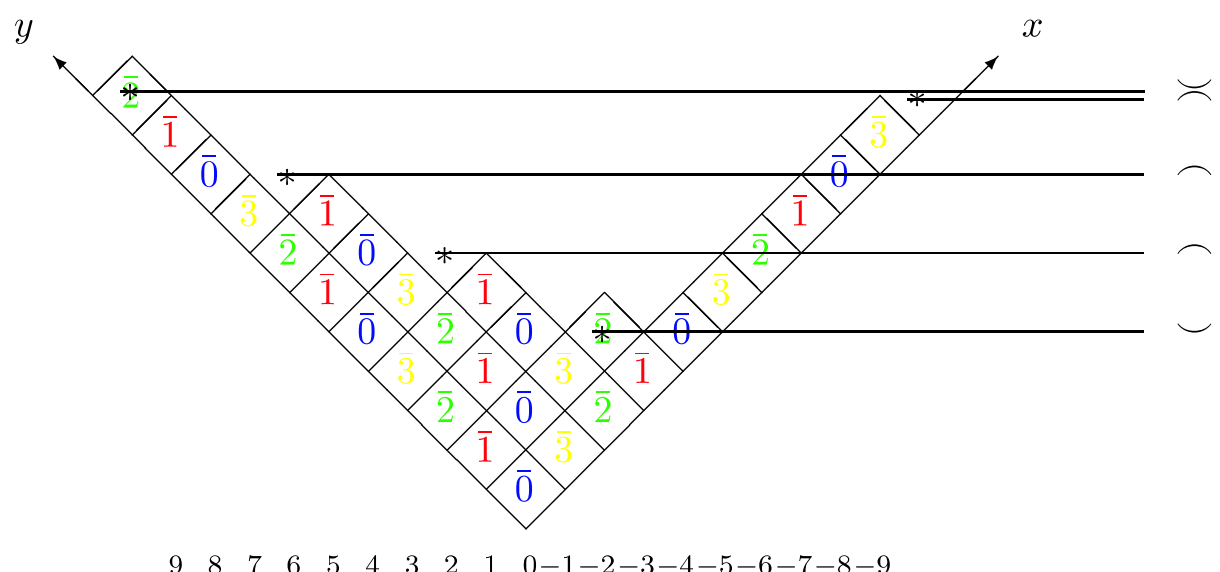

Figure 3. The partition $\lambda=(11,7,4,2,1,1,1,1,1,1)$ is an element of $B^{H}$ for $\widehat{\mathfrak{s l}}_{4}$, since no box $b$ in $\lambda$ is $A^{H}$-illegal. The color $\bar{c}(b)$ of each box $b$ is written inside $b$. We demonstrate the calculation of $\tilde{f}_{\overline{2}}(\lambda)$. The string of brackets $S_{\overline{2}}^{H}(\lambda)$ has a "(" for each $\overline{2}$-addable box and a ")" for each $\overline{2}$-removable box, ordered from left to right lexicographically, first by decreasing height $h(b)$, then by decreasing content $c(b)$. The result is shown on the right of the diagram (rotate the page 90 degrees counter clockwise). Thus $\tilde{f}_{\overline{2}}(\lambda)$ is the partition obtained by adding the box with coordinates $(x, y)=(10.5,0.5)$. The map $\Psi$ from Section 5 takes $\lambda$ to $Y_{\overline{3}, 11} Y_{\overline{2}, 8} Y_{\overline{2}, 6} Y_{\overline{3}, 5} Y_{\overline{1}, 5} Y_{\overline{2}, 10} Y_{\overline{2}, 12}^{-1} Y_{\overline{1}, 9}^{-1} Y_{\overline{1}, 7}^{-1} Y_{\overline{2}, 6}^{-1} Y_{\overline{3}, 11}^{-1}$. After reordering and simplifying, this becomes $Y_{\overline{2}, 12}^{-1} Y_{\overline{2}, 10} Y_{\overline{1}, 9}^{-1} Y_{\overline{2}, 8} Y_{\overline{1}, 7}^{-1} Y_{\overline{1}, 5} Y_{\overline{3}, 5}$. The string of brackets $S_{\overline{2}}^{M}(\Psi(\lambda))$ is the same as the string of brackets $S_{\overline{2}}^{H}(\lambda)$, except that a canceling pair () has been removed. The condition that $\lambda$ has no $A^{H}$-illegal boxes implies that $S_{\bar{\imath}}^{H}(\lambda)$ and $S_{\bar{l}}^{M}(\Psi(\lambda))$ are always the same up to removing pairs of canceling brackets, which is essentially the proof that $\Psi$ is an isomorphism.

- Either $(i)$ : $A_{\bar{\imath}+\overline{1}}(\mu) \backslash A_{\bar{\imath}+\overline{1}}(\lambda)=b^{\prime}$ and $R_{\bar{\imath}+\overline{1}}(\lambda)=R_{\bar{\imath}+\overline{1}}(\mu)$ for some box $b^{\prime}$ with $h\left(b^{\prime}\right)=$ $h(b)+1$, or $(i i): R_{\bar{\imath}+\overline{1}}(\lambda) \backslash R_{\bar{\imath}+\overline{1}}(\mu)=b^{\prime}$ and $A_{\bar{\imath}+\overline{1}}(\lambda)=A_{\bar{\imath}+\overline{1}}(\mu)$ for some box $b^{\prime}$ with $h\left(b^{\prime}\right)=h(b)-1$.

- Either $(i)$ : $A_{\bar{\imath}-\overline{1}}(\mu) \backslash A_{\bar{\imath}-\overline{1}}(\lambda)=b^{\prime \prime}$ and $R_{\bar{\imath}-\overline{1}}(\lambda)=R_{\bar{\imath}-\overline{1}}(\mu)$ for some box $b^{\prime \prime}$ with $h\left(b^{\prime \prime}\right)=$ $h(b)+1$, or $(i i): R_{\bar{\imath}-\overline{1}}(\lambda) \backslash R_{\bar{\imath}-\overline{1}}(\mu)=b^{\prime \prime}$ and $A_{\bar{\imath}-\overline{1}}(\lambda)=A_{\bar{\imath}-\overline{1}}(\mu)$ for some box $b^{\prime \prime}$ with $h\left(b^{\prime \prime}\right)=h(b)-1$.

By the definition of $\Psi$, this implies

$$
\Psi(\mu)=Y_{\bar{\imath}, h(b)-1}^{-1} Y_{\bar{\imath}, h(b)+1}^{-1} Y_{\bar{\imath}+\overline{1}, h(b)} Y_{\bar{\imath}-\overline{1}, h(b)} \Psi(\lambda)=A_{\bar{\imath}, h(b)}^{-1} \Psi(\lambda) .
$$

Lemma 5.3. Let $\lambda \in B^{H}$, and choose $b \in A_{\bar{\imath}}(\lambda), b^{\prime} \in R_{\bar{\imath}}(\lambda)$. Then

(i) $h(b) \neq h\left(b^{\prime}\right)+1$.

(ii) If $h(b)=h\left(b^{\prime}\right)$ then $c\left(b^{\prime}\right)>c(b)$, so $b^{\prime} \succ_{A^{H}} b$.

(iii) $b \succ_{A^{H}} b^{\prime}$ if and only if $h(b)-1 \geq h\left(b^{\prime}\right)+1$.

(iv) Assume $h(b)-1=h\left(b^{\prime}\right)+1$. If $c \in A_{\bar{\imath}}(\lambda)$ satisfies $b \succ_{A^{H}} c \succ_{A^{H}} b^{\prime}$, then $h(c)=h(b)$.

$(v)$ Assume $h(b)-1=h\left(b^{\prime}\right)+1$. If $c \in R_{\bar{\imath}}(\lambda)$ satisfies $b \succ_{A^{H}} c \succ_{A^{H}} b^{\prime}$, then $h(c)=h\left(b^{\prime}\right)$.

Proof. By the definitions of $A_{\bar{\imath}}(\lambda)$ and $R_{\bar{\imath}}(\lambda), b$ and $b^{\prime}$ cannot lie in either the same row or the same column, which implies that there is a unique box $a$ in $\lambda$ which shares a row or column with each of $b, b^{\prime}$. It is straightforward to see that if $(i)$ or $(i i)$ is violated then this $a$ is $A^{H}$-illegal (see Fig. 4).

To see part (iii), recall that by Lemma $4.4, b \succ_{A^{H}} b^{\prime}$ if and only if $h(b)>h\left(b^{\prime}\right)$ or both $h(b)=h\left(b^{\prime}\right)$ and $c(b)>c\left(b^{\prime}\right)$. This order agrees with the formula in part $(i i i)$, since parts $(i)$ and $(i i)$ eliminate all cases where they would differ. 


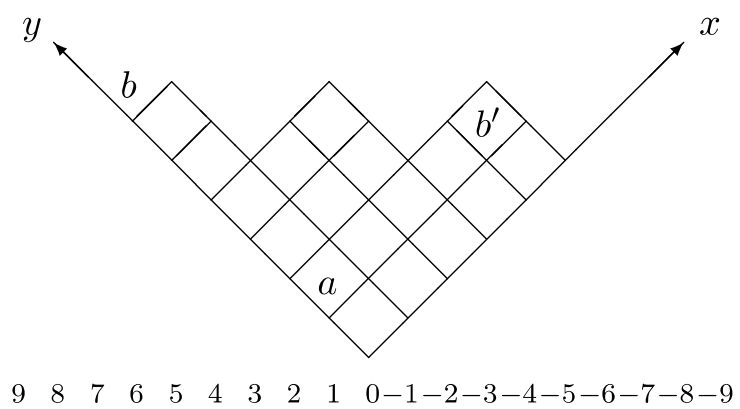

Figure 4. The hook defined by $b \in A_{\bar{\imath}}(\lambda)$ and $b^{\prime} \in R_{\bar{\imath}}(\lambda)$. There will always be a unique box $a$ in $\lambda$ which is in either the same row or the same column as $b$ and also in either the same row or the same column as $b^{\prime}$. Taking $n=3$, we have $b \in A_{\overline{0}}(\lambda)$ and $b^{\prime} \in R_{\overline{0}}(\lambda)$. Then $\operatorname{hook}(a)=9=3 \times 3$ and $\operatorname{arm}(a)=4=A_{3}^{H}$, so $a$ is $A^{H}$-illegal. It is straightforward to see that in general, if either $(i): h(b)=h\left(b^{\prime}\right)+1$ or $(i i): h(b)=h\left(b^{\prime}\right)$ and $c(b)>c\left(b^{\prime}\right)$, then the resulting hook is $A^{H}$-illegal, and hence $\lambda \notin B^{H}$.

Part (iv) and (v) follow because any other $c \in A_{\bar{\imath}}(\lambda) \cup R_{\bar{\imath}}(\lambda)$ with $b \succ_{A^{H}} \quad c \succ_{A^{H}} b^{\prime}$ would violate either $(i)$ or $(i i)$.

Proof of Theorem 5.1. Fix $\lambda \in B^{H}$ and $\bar{\imath} \in \mathbb{Z} / n \mathbb{Z}$. Let $S_{\bar{\imath}}^{M}(m)$ denote the string of brackets used in Section 3 to calculate $\tilde{e}_{\bar{\imath}}^{M}(m)$ and $\tilde{f}_{\bar{\imath}}^{M}(m)$. Let $S_{\bar{\imath}}^{H}(\lambda)$ denote the string of brackets used in Section 4 to calculate $\tilde{e}_{\bar{\imath}}^{H}(\lambda)$ and $\tilde{f}_{\bar{\imath}}^{H}(\lambda)$, and define the height of a bracket in $S_{\bar{\imath}}^{H}(\lambda)$ to be $h(b)$ for the corresponding box $b \in A_{\bar{\imath}}(\lambda) \cup R_{\bar{\imath}}(\lambda)$.

By Lemma 5.3 parts $(i v)$ and $(v)$, for each $k \geq 1$, all "(" in $S_{\bar{\imath}}^{H}(\lambda)$ of height $k+1$ are immediately to the left of all ")" of height $k-1$. Let $T$ be the string of brackets obtained from $S_{\bar{\imath}}^{H}(\lambda)$ by, for each $k$, canceling as many "(" of height $k+1$ with ")" of height $k-1$ as possible. Notice that one can use $T$ instead of $S_{\bar{l}}^{H}(\lambda)$ to calculate $\tilde{e}_{\bar{l}}^{H}(\lambda)$ and $\tilde{f}_{\bar{\imath}}^{H}(\lambda)$ without changing the result.

By the definition of $\Psi$, it is clear that

(i) The "(" in $T$ of height $k+1$ correspond exactly to the factors of $Y_{\bar{\imath}, k}$ in $\Psi(\lambda)$.

(ii) The ")" in $T$ of height $k-1$ correspond exactly to the factors of $Y_{\bar{\imath}, k}^{-1}$ in $\Psi(\lambda)$.

Thus the brackets in $T$ correspond exactly to the brackets in $S_{\bar{\imath}}^{M}(\Psi(\lambda))$. Furthermore, Lemma 5.3 part (iii) implies that these brackets occur in the same order. The theorem then follows from Lemma 5.2 and the definitions of the operators (see equations (2) and (3)).

Corollary 5.4. For any $n, \mathcal{M}\left(Y_{\overline{0}, 0}\right)$ is a copy of the fundamental crystal $B(\Lambda(0))$ for $\widehat{\mathfrak{s l}}_{n}$.

Proof. This follows immediately from Theorem 5.1, since, by Theorem $4.1, B^{H}$ is a copy of the crystal $B\left(\Lambda_{0}\right)$.

\section{Questions}

Question 1. Nakajima originally developed the monomial crystal using the theory of $q$-characters from [4]. Can this theory be modified to give rise to any of Fayers' other crystal structures? One may hope that this would help explain algebraically why these crystal structures exist.

Question 2. In [11], Kim considers a modification to the monomial crystal developed by Kashiwara [10]. She works with more general integral highest weight crystals, but restricting her results to $B\left(\Lambda_{0}\right)$ one finds a natural isomorphisms between this modified monomial crystal and 
the Misra-Miwa realization. The Misra-Miwa realization corresponds to one case of Fayer's partition crystal, but not the one studied in Section 4.3. In [10], there is some choice as to how the monomial crystal is modified. Do other modifications also correspond to instances of Fayers crystal? Which instances of Fayers' partiton crystal correspond to modified monomial crystals (or appropriate generalizations)?

Question 3. The monomial crystal construction works for higher level $\widehat{\mathfrak{s l}}_{n}$ crystals. There are also natural realizations of higher level $\widehat{\mathfrak{s l}}_{n}$ crystals using tuples of partitions (see [3, 7, 8, 14]). Is there an analogue of Fayers' construction in higher levels generalizing both of these types of realization?

\section{Acknowledgments}

We thank Chris Berg, Matthew Fayers, David Hernandez and Monica Vazirani for interesting discussions. This work was supported by NSF grant DMS-0902649.

\section{References}

[1] Berg C., ( $\ell, 0)-J M$ partitions and a ladder based model for the basic crystal of $\widehat{\mathfrak{s l}}_{\ell}$, arXiv:0901.3565.

[2] Fayers M., Partition models for the crystal of the basic $U_{q}\left(\widehat{\mathfrak{s l}}_{n}\right)$-module, J. Algebraic Combin., to appear, arXiv:0906.4129.

[3] Foda O.,, Leclerc B., Okado M., Thibon J.-Y., Welsh T.A., Branching functions of $A_{n-1}^{(1)}$ and Jantzen-Seitz problem for Ariki-Koike algebras, Adv. Math. 141 (1999), 322-365, q-alg/9710007.

[4] Frenkel E., Reshetikhin N., The $q$-characters of representations of quantum affine algebras and deformations of $\mathcal{W}$-algebras, in Recent Developments in Quantum Affine Algebras and Related Topics (Raleigh, NC, 1998), Contemp. Math., Vol. 248, Amer. Math. Soc., Providence, RI, 1999, 163-205, math.QA/9810055.

[5] Hernandez D., Nakajima H., Level 0 monomial crystals, Nagoya Math. J. 184 (2006), 85-153, math.QA/0606174.

[6] Hong J., Kang S.-J., Introduction to quantum groups and crystal bases, Graduate Studies in Mathematics, Vol. 42, Amer. Math. Soc., Providence, RI, 2002.

[7] Jimbo M., Misra K.C., Miwa T., Okado M., Combinatorics of representations of $\mathrm{U}_{q}(\widehat{\mathfrak{s l}}(n))$ at $q=0$, Comm. Math. Phys. 136 (1991), 543-566.

[8] Kang S.-J., Lee H., Higher level affine crystals and Young walls, Algebr. Represent. Theory 9 (2006), 593632, math.QA/0310430.

[9] Kashiwara M., On crystal bases, in Representations of Groups (Banff, AB, 1994), CMS Conf. Proc., Vol. 16, Amer. Math. Soc., Providence, RI, 1995, 155-197.

[10] Kashiwara M., Realizations of crystals, in Combinatorial and Geometric Representation Theory (Seoul, 2001), Contemp. Math., Vol. 325, Amer. Math. Soc., Providence, RI, 2003, 133-139, math.QA/0202268.

[11] Kim J.-A., Monomial realization of crystal graphs for $U_{q}\left(A_{n}(1)\right)$, Math. Ann. 332 (2005), 17-35.

[12] Misra K., Miwa T., Crystal base for the basic representation of $U_{q}\left(\widehat{\mathfrak{s l}}_{n}\right)$, Comm. Math. Phys. 134 (1990), $79-88$.

[13] Nakajima H., $t$-analogs of $q$-characters of quantum affine algebras of type $A_{n}, D_{n}$, in Combinatorial and Geometric Representation Theory (Seoul, 2001), Contemp. Math., Vol. 325, Amer. Math. Soc., Providence, RI, 2003, 141-160, math.QA/0204184.

[14] Tingley P., Three combinatorial models for $\widehat{\mathrm{sl}}_{n}$ crystals, with applications to cylindric plane partitions, Int. Math. Res. Not. IMRN 2008 (2008), no. 2, Art. ID rnm143, 40 pages, math.QA/0702062. 